PA - 1 - 1

\section{Reducing Medical Student Performance Anxiety through the Pre-clerkship Residency Exploration Program (PREP)}

Alysha Roberts Dalhousie University, Kavita Raju Dalhousie University, Thomas Sebastian Haupt Dalhousie University, Todd Dow Dalhousie University, Mike Smyth Dalhousie University, J Thomas Toguri Dalhousie University

Background/Purpose: Medical students face numerous stressors during their pre-clinical years, including developing confidence in their skills, knowledge and deciding which specialty to pursue. For those interested in specialties with limited integration into the traditional pre-clinical curriculum, or students who still have a broad range of interests at the end of their pre-clinical studies, a two-week Pre-clerkship Residency Exploration Program (PREP) could be a useful tool to reduce career-decision-making anxiety. Objectives: To assess whether PREP, a two-week intensive elective for second year medical students, can be effective in reducing performance anxiety and stress related to the transition into a clinical learning environment.

Methods: 37 second year medical students completed pre- and post-program surveys that assessed, on a Likert-scale, their level of performance anxiety during clinical electives, as well as their anxiety oriented towards future career decisions (i.e., clerkship, specialty selection, and residency match).

Results: Of those who participated, performance anxiety was low at baseline and was unchanged by the end of PREP ( $p$-value $=0.29$ ). However, there was a significant decrease in stress surrounding exposure to enough specialties to make an informed career choice ( $p$-value < 0.001).

Conclusion: While this cohort of participants were not stressed about their clinical performance, they are anxious to explore as many specialties as possible during their pre-clinical years to help make informed career decisions. PREP may serve as an effective tool to expose medical students to a high volume of specialties and reduce the stress associated with transitioning into a clinical learning environment.

\section{PA - 1 - 2 \\ Do offers of admission to regional medical campuses impact an applicant's decision to decline their acceptance to medical school?}

Rosalind Bihun McMaster University, Margo Mountjoy McMaster University, Andrew Costa McMaster University, Graham Campbell McMaster University

Background/Purpose: While there is extensive published research describing how to best select medical school applicants, there is a paucity of research on how applicants choose a medical school if they have multiple offers of admission. The aim of this study was to assess if an offer of admission to a regional medical campus (RMC) had any impact on an applicant's choice to decline their offer.

Methods: This study analyzed surveys completed by successful medical school applicants who declined their offer of admission to McMaster University. Survey data from 2013 to 2018 were analyzed. Quantitative data assessed applicants' rankings of preselected factors. Data was pooled and descriptive statistics were generated. Annual decline percentages for each campus were then compared. Qualitative data in the form of survey comments were analyzed using an applied thematic analysis framework. Responses were analyzed for similar thematic elements.

Results: Quantitative analysis revealed that the type of campus to which a student was offered admission was one of the least influential factors on an applicant's decision to decline their offer. Additionally, there was no significant difference between campus specific decline percentages as calculated by a one-way analysis of variance. Of the survey comments analyzed in the qualitative analysis only $11.8 \%$ related to RMCs. Themes seen within 
these comments were community, transportation, and opportunities.

Conclusion: Data analysis demonstrated that an applicant's decision to decline an offer of admission to medical school is not influenced by the campus that they were assigned to.

\section{PA - 1 - 3 \\ Increasing Diversity Amongst Future Physicians - MD Admissions Initiative for Diversity and Equity}

Emily Fong University of Alberta, Alex Wong University of Alberta, Tibetha Kemble University of Alberta

Background/Purpose: According to the Association of Faculties of Medicine of Canada (AFMC), medical schools have a mandate to be socially accountable and train future physicians to meet the needs of the communities they serve. It is a growing concern that current medical school cohorts do not reflect the diversity of the Canadian population. In particular, students from low socioeconomic (SES) and First Nations, Metis, and Inuit backgrounds are underrepresented among Canadian medical students. This has important implications given that physicians are more likely to practice in their communities of origin. Significant financial and social barriers exist throughout medical school admission that disadvantage students from marginalized backgrounds - one of which being the Medical College Admissions Test (MCAT). This necessitates that efforts are made to address these barriers to encourage diverse applicants to apply for medical school.

Summary of the Innovation: The MD Admissions Initiative for Diversity and Equity (MD AIDE) is a student-led initiative at the University of Alberta that provided 33 students from low SES or Indigenous backgrounds with free tutoring and resources for the MCAT and along with mentorship from medical students. 3-hour MCAT preparation sessions led by medical students were held twice a week from May July 2018. Classes were interactive and focussed on questions and test-taking strategies. To address the importance of social networks, each student was also matched to a medical student to assist them with career planning. For evaluation purposes, midpoint and final surveys were conducted to gather quantitative data on targeted outcomes. Interviews were scheduled with all willing participants to gather qualitative data on the effectiveness of the initiative.

Conclusion: of the MD AIDE participants, a statistically significant number have completed the MCAT and applied to a Canadian medical school. 96\% of MD AIDE participants would recommend this program to their peers. MD AIDE is a one piece of a larger systemic change that is required to build diversity and equity within medical schools

\section{PA - 1 - 4 \\ Improving medical student education in pediatrics through participation in Teddy Bear Clinics}

Stephanie Chan McMaster University, Nathasha Dias
McMaster University, Amanda Leslie McMaster
University

Background/Purpose: In McMaster University's medical curriculum, preclerkship students have little exposure working with pediatric populations, and limited time spent learning about important child health topics. This quality gap was identified through a needs assessment survey completed by students spanning 3 cohorts.

Summary of the Innovation: Teddy Bear Clinics (TBCs) are an interactive opportunity for medical students to guide children in role playing medical scenarios using teddy bears. Children work through clinical situations including doctor visits and health promotion stations. Stations are run by medical student volunteers who are required to attend a Public Health Niagara teaching session. While research exists on the benefits of TBCs in alleviating children's fears about healthcare, there is currently no evidence on their role in medical education. Our project investigates the use of TBCs in educating medical students on pediatric health and improving comfort in interacting with children. Quality improvement methodology was employed with PlanDo-Study-Act cycles. Pre- and post-surveys were done by participants in TBCs and included questions on 
perception of their pediatrics knowledge and comfort working with children.

Conclusion: Pre-surveys highlighted the need for additional training in pediatrics for preclerkship students. Post-surveys showed improvement in students' feelings of preparedness, confidence, comfort working the the pediatric population, and increased breadth of knowledge in high yield pediatric health topics. We plan on expanding this initiative to all McMaster campuses. A curriculum proposal has been submitted for consideration of incorporating pediatric public health teaching and TBCs into the existing curriculum.

\section{PA - 1 - 5}

Development and implementation of an Intellectual and Developmental Disability curriculum for first-year medical students at the University of Toronto

Maggie McCann University of Toronto, Kristen McFadyen University of Toronto, Olivia Spohn University of Toronto, Janet Vogt Surrey Place Centre, Barry Isaacs Surrey Place Centre, Alvin Loh Surrey Place Centre, Fok-Han Leung University of Toronto

Background/Purpose: There is a gap in medical education regarding intellectual and developmental disabilities (IDD), causing a lack of confidence and competence among physicians caring for these patients. For people with IDD, clinical exposures can be distressing, which may result in negative attitudes and failure to recognize the humanity of these patients by clinicians. Therefore, the new preclerkship curriculum at the University of Toronto focuses on enabling students to develop a broader understanding of IDD in a community context.

Summary of the Innovation: The curriculum has three components: (i) pre-community agency visit tutorial, (ii) community agency visit, and (iii) reflection and service access support tutorial. The curriculum is centered around preparing students for the community agency visit, and facilitating a small group reflection session following the visit. The visits are designed to provide students with the opportunity to gain experience communicating with people with IDD in a non-clinical setting, to better understand the variety of communities in which people live, and to learn how to connect people with intellectual and developmental disabilities specifically with community resources.

Conclusion: The development of this curriculum fills a gap identified at not only the University of Toronto, but one that has been identified by medical schools worldwide. Through working with Surrey Place Centre, a multi-service Toronto-based organization that supports individuals with IDD, and other community agencies in the Greater Toronto Area, we have been met with overwhelming support for this initiative. The community visits and associated tutorials are being implemented for first year medical students starting in 2018.

\section{PA - 1 - 6 \\ Development of spaced repetition materials for undergraduate medical histopathology education}

Jeremy Dick University of British Columbia, Karen Pinder University of British Columbia

Background/Purpose: The purpose of this project was to generate interest in, and facilitate learning of, histology and pathology through the development of educational materials for the undergraduate medical school curriculum at the University of British Columbia (UBC). These materials were designed to utilize a spaced repetition format of learning to improve student recall of histopathological concepts of medicine.

Summary of the Innovation: Electronic flashcards were developed that were based around the UBC medical school's histopathology curriculum to facilitate recall of, and generate student interest in, histology and pathology. The content incorporated both annotated digital slides of histology sections from the medical school curriculum and concepts inspired by an online pathology course. The flashcards were designed using Anki computer software and provided students with an effective means of developing long-term understanding via active recall and spaced repetition. Ultimately, the histopathology flashcards were compiled and distributed to students through the medical school's 
histology website and a survey was available for students to provide feedback on the effectiveness of the materials.

Conclusion: Nearly nine hundred flashcards were created for the UBC medical histopathology curriculum. A survey was distributed to assess the utility of the materials for helping students prepare for exams and for improving their general histology/pathology knowledge. Of the responses, $89 \%$ found the content helpful for exam preparation and improving knowledge. This initiative is efficacious in enhancing student learning and promoting core foundational medical sciences content and it will be of interest to students and faculty in medical and allied fields.

\section{PA - 1 - 7 Exploring international experiences of climate change in medical education}

\section{George T. Kitching Western University, Adrina Zhong Western University, Orianna Mak McMaster University, Marissa Ley Dalhousie University, Farnaz Javadian University of British Columbia}

Background/Purpose: Climate change is considered by many to be the greatest threat to global health of the 21st century. Yet, medical trainees around the world receive limited education on the health impacts of climate change. At the August 2018 General Assembly of the International Federation of Medical Students Associations (IFMSA), we explored the views and experiences of medical students' education of climate change within their formal medical curriculum.

Methods: We recruited medical students at the IFMSA General Assembly. Semi-structured interviews were conducted with participants in person or over email after the general assembly. In-person interviews were audio-recorded and transcribed verbatim. We used thematic analysis to identify key overarching concepts that emerged from the interviews.

Results: We interviewed 18 medical students from 16 countries including: Algeria, Australia, Austria, Belgium, Germany, Hong Kong, Japan, Jordan, Luxembourg, Norway, Portugal, Trinidad and Tobago,
Singapore, South Korea, Switzerland, and United Kingdom. We identified 3 main themes: 1) Climate change is generally not taught within formal medical curriculum globally; students learn from informal opportunities and extracurricular involvement. 2) Students are concerned about how climate change is affecting their countries and believe health impacts should be incorporated into medical education. 3) Students believe physicians have the opportunity to be leaders on multiple levels (organizational, clinical, individual) in engaging the general public in mitigating and adapting to effects of climate change.

Conclusion: There is demand and support from medical students around the world for implementation of medical education regarding the health impacts of climate change.

\section{PA - 1 - 8 Standing Up to Block: Validating a Novel Ob/Gyn LIC Curriculum at the University of Toronto}

Alexandra Davidson University of Toronto, Rajiv Shah University of Toronto, Anita Shah McMaster University, Lindsay Shirreff University of Toronto, Eliane Shore University of Toronto

Background/Purpose: Longitudinal Integrated Clerkship (LIC) was introduced at the University of Toronto in 2014. A novel Ob/Gyn curriculum was designed to incorporate both ambulatory and inpatient experiences into a new formal teaching program. Compared to the Block curriculum, one difference is that LIC students are assessed midway through the academic year, whereas Block students are assessed at the end of their six-week rotation. However, both streams of students take equivalent examinations. The purpose of this study was to assess the validity of our Ob/Gyn LIC when compared to the existing Block clerkship.

Methods: Written, oral, and clinical examination scores from September 2014 to March 2018 were compared between LIC and Block students. Specifically, grade comparisons were made between students in the same academic year, between years, and between hospital sites $(n=4)$. 
Results: Eighty-nine LIC students and 797 Block students were compared. There were no statistical differences between LIC and Block clinical and oral examination scores (Clinical: $81.0 \%$ vs $81.2 \%$, Oral: $83.9 \%$ vs $84.4 \%, p>0.05$ ). However, Block students did perform significantly better on written exams $(83.0 \%$ vs $79.5 \%, p<0.05)$.
Conclusion: Our LIC curriculum prepares students as well as their Block peers for assessments, but students in the LIC program have the added advantage of longitudinal relations with faculty and patients. Thus, we have validated our Ob/Gyn LIC program and anticipate that our curriculum can be appropriately modelled at other teaching institutions. 
PA - 2 - 1

\section{The Community Health and Social Medicine Incubator (CHASM): A Pilot of Student Led Social Change}

David-Dan Nguyen McGill, Julie de Meulemeester McGill, Kacper Niburski McGill, Gordon Best McGill, Koray Demir McGill

Background/Purpose: Medical schools across Canada have incorporated service-learning into their curricula. Service-learning allows students to dismantle the perceived separation of work and civic contributions at the root of many health inequities. We adopt a corporate incubator model for community health needs as an approach to innovative service learning.

Summary of the Innovation: CHASM was founded by McGill medical students in June 2017 to improve the health outcomes of local, historically marginalized communities. CHASM helps students partner with a community organization, form an interprofessional team, and develop a project to address the population's self-identified social determinants of health. The end goal is for the project to be entirely handed over to the community. The recruited projects are given a framework to scale their ideas, which includes: 1 . Mentoring by business and public health experts, largely faculty at McGill University; 2. A social entrepreneurship curriculum with bimonthly, interactive workshops; 3. Funding; 4. A network that supports and publicizes the projects. The inaugural cohort is composed of three teams: Monthly Dignity, which provides feminine hygiene products to homeless shelters; Supporting Young Black Students, which mentors Black Montreal youth to facilitate their admission to university health programs; and Community Ambassadors to Conquer HPV, which provides HPV vaccines and pap smears to underprivileged women.

Conclusion: This unprecedented educational opportunity allows students to partner with local communities to sustainably address their social determinants of health, while simultaneously acquiring project development and advocacy skills. Successful in its first year, we envision the CHASM Incubator model as an integral part of Canadian medical education.

\section{PA - 2 - 2 \\ Naloxone kits at AEDs: A case-study of medical student driven advocacy in London, Ontario}

Eric Mitchell Western University, James Riggs Western University, Rebecca Barnfield Western University, Trystan Nault Western University, Rajas Tipnis Western University

Background/Purpose: Physicians have the capacity and responsibility to act as advocates for their patients on a daily basis. Increasingly, physicians are effectively advocating for change in healthcare systems, and more broadly in society as a whole. As future physicians, medical students are expected to develop advocacy skills that will allow them to fill these roles in the future. However, opportunities to learn these skills are limited in the undergraduate medical curricula.

Summary of the Innovation: A group of medical students, collectively known at the Schulich Political Advocacy Committee (PAC) lobbied London's city councillors to install two naloxone kits at each automated external defibrillator (AED) located in cityowned public buildings. Naloxone reverses opioid poisoning and having it widely available reduces mortality associated with opioid poisoning. In October 2017, PAC leadership developed briefs on three potential advocacy topics and solicited feedback from medical students via an online survey. The topic of greatest interest was the placement of naloxone kits at each city-owned AED. In May 2018, first, second, and third year medical students at the Schulich School of Medicine \& Dentistry participated in the PAC's 2018 Lobby Day initiative. Each student attended a four-hour training session and at least one 
city councillor meeting. Subsequent council votes and committee meetings were attended by PAC executive members, culminating in a motion being unanimously approved by council.

Conclusion: Medical students are willing and able to successfully advocate for significant change in their communities. With sufficient organization and leadership, the Schulich PAC made a substantial impact on their community, and strives to continue this work in the years to come.

\section{PA - 2 - 3 \\ Medical Student Reflections on a Personalized Resource List}

Leshawn Benedict University of Toronto, Kevin Si University of Toronto, Fok-Han Leung University of Toronto

Background/Purpose: Networking among physicians is an under-emphasized skill that can take years of experience to build upon and execute successfully. Today, physician's create vast connections with community organizations, healthcare practitioners, and other service workers to provide the best care for their patients. Recognizing the importance, the Faculty of Medicine at the University of Toronto introduced a Personalized-Resource List (PeRL) to first-year medical students. Students were tasked with building a contact list to support their future practice. Examples of contacts included healthcare practitioners, and community organizations. PeRL is a longitudinal assignment that was designed to adapt to the student's medical training over the course of their career and to provide medical students with the skills necessary to develop and maintain professional relationships. Strengthening this skill would encourage future physicians to look beyond the scope of their own practice and provide comprehensive care. This study aims to collect feedback from students to improve the next iteration of the assignment and to assess the impact of PeRL in medical education.

Summary of the Innovation: We conducted 9 semistructured interviews to elicit perspectives of firstyear medical students regarding the PeRL. The interviews were analyzed using thematic analysis to identify common themes.

Conclusion: The majority of students recognized PeRL as an important aspect of their education. Formation of their identity as a physician, relationship development with patients, and meshing with the rest of the curriculum were important to students. In the next iteration, it was suggested to have more reallife examples of network building, and to develop more formal evaluations.

\section{PA - 2 - 4}

\section{Tips: A Guideline to Establishing a Successful Medical Education Program}

Thomas Haupt Dalhousie University, Todd Dow Dalhousie University, Mike Smyth Dalhousie University, Alysha Roberts Dalhousie University, Kavita Raju Dalhousie University, Tom Toguri Dalhousie University, Anna MacLeod Dalhousie University

Background/Purpose: The field of medical education is constantly evolving and integrating new techniques. Unique programs and initiatives are discussed, but individuals often struggle to start or establish their idea. The 12 tips provided here offer a guide on how to take an educational program idea from inception and bring it to fruition.

Summary of the Innovation: This guide provides the following 12 tips, with in-depth detail on how to initiate a successful medical education program.

1: Ensure your idea is novel and of value

2: Assemble a team that works well together but differs in their strengths

3: Establish your short and long-term goals early but do not hesitate to revise

4: Consult and obtain buy-in from your institution

5: Create a win-win situation for you and the institution

6: Identify administrative obstacles quickly

7: Create a succession plan

8: Establish a means of communication with participants and medical departmental heads 
9: To assess impact of your program, do research

10: Prepare for the transition of leadership

11: Quality assurance is an ethical imperative

12: Growth and development spreads a good idea from the few to the many

Conclusion: Medical education aims to improve the well-being of societies through improved training of medical practitioners. This is accomplished by improvements in how we train the healthcare professionals of tomorrow. The 12 tips provided here offer a guide that is transferable and applicable to anyone wishing to initiate an educational program.

\section{PA - 2 - 5}

\section{An Interactive iBook for University of Toronto Anesthesia Medical Students}

Alex Sapa University of Toronto, Ahtsham Niazi University of Toronto, Clyde Matava University of Toronto

Background/Purpose: Many medical school programs are looking to e-learning for teaching due to the rapid increase in the use of smartphones, tablets and portable computers. E-learning allows an increased access to students, allows students to learn remotely and reduces program costs. iBooks have been used as e-learning tools with students praising their interactivity, multimedia, and logical presentation. 1 In addition, studies show equivalent results on standardized testing when using elearning. 2

Summary of the Innovation: Three years ago, the manual for medical students attending the anesthesia clerkship course at the University of Toronto ( $\mathrm{U}$ of $\mathrm{T}$ ) was converted to an iBook. This was implemented in order to make the rotation paperless and to align with the flipped classroom model which was started five years ago. The iBook allowed readers to highlight, bookmark and take notes, and allowed the authors to embed figures and videos directly into the text. The iBook was then made available for all $U$ of $T$ medical students on both android and iOS platforms as well as on iTunes. This change to an iBook format led to over $\$ 3500$ in savings per year, while providing students with additional access to interactive charts and content. The iBook to date has been downloaded 1,041 times worldwide. USA and Canada have contributed to most of the book downloads (863), with the remainder being from Europe, Latin America, and Asia.

Conclusion: The anesthesia iBook has been very well accepted by the students at $U$ of $T$. Challenges of the iBook have been a call from a minority of students for a printable resource and a preference for the portable document format. Future questionnaires will be used to identify common themes of learning in students more familiar with an e-learning curriculum.

\section{PA - 2 - 6}

Development and evaluation of a new interactive genetic education module as a teaching tool in the Division of Clinical and Metabolic Genetics at the Hospital for Sick Children

Tina (Shijie) Zhou Western University, Lucie Dupuis University of Toronto, Cheryl Cytrynbaum University of Toronto, Roberto Mendoza-Londono University of Toronto

Background/Purpose: Knowledge of genetics is needed by health professionals to practise in the current genomic era. The Division of Clinical and Metabolic Genetics has limited teaching resources to meet the increasing demand from trainees seeking electives. Currently, much of the teaching is didactic in nature or relies upon review of written materials. The benefits of e-learning in medical education are well recognized. The Division therefore undertook the development of a series of online education modules to teach the foundation of a genetic assessment to complement clinical teaching. This will not only address resource constraints, but also promote independent learning, thereby increasing teaching efficiency while ensuring quality care.

Summary of the Innovation: An e-learning module was created using Articulate Storyline 2 and the principles of case-based learning. The module focuses on how to take a family history (specific to a genetic assessment), and how to draw and interpret pedigrees. The module incorporates interactive 
graphics and quizzes to engage the learners, and provides detailed feedbacks on user responses.

Conclusion: Surveys on module usability and user satisfaction, as well as pre-module and post-module quizzes were completed by trainees rotating through the Division $(n=11)$. System usability scale is rated at 73.75 (out of 100 ), while user satisfaction is reported as 4.3 (out of 5). Pre-module and post-module quizzes revealed an average increase of $33 \%$ in score after completion of the module $(n=4)$. We will incorporate the E-learning module as part of the curriculum within the Division, and create more modules for further educational topics.

\section{PA - 2 - 7}

\section{Preparing for Surgery Observerships during Medical School: A Cross-Sectional Study}

Arielle Brickman Queen's University, Vincent Wu University of Toronto, Boris Zevin Queen's University

Background/Purpose: students have traditionally used a variety of methods to prepare for observerships - clinical experiences that provide exposures to different specialties and help guide career choices. We aimed to evaluate the types and number of resources utilized by pre-clerkship medical students at a Canadian medical school in their preparation for surgery observerships.

Methods: Pre-clerkship medical students were recruited on a voluntary basis to complete an online survey. The survey collected both qualitative and quantitative data related to the observership experience with the use of multiple choice, free-text, and Likert-type scales. Results were analyzed using descriptive statistics.

Results: Of the 64 respondents (64/202), 62.5\% were interested in a career in surgery and $82.8 \%$ participated in at least one surgery observership. Common reasons for participating in a surgery observership were: "may not be an opportunity I have in the future" (28.3\%) and "rule in surgery as a career" (27.3\%). Students spent on average 0.98 hours (SD $0.77)$ preparing for an observership. Most frequently used resources included class notes $(22.6 \%)$ and Youtube videos (20.2\%). The majority (96.9\%) of participants noted that preparing resulted in an overall positive observership experience. However, $75.44 \%$ noted that resources provided and instructions were lacking.

Conclusion: Preparing for surgery observerships resulted in a more positive learning experience. Class notes and Youtube videos were the most frequently used resources; however, formal instructions on how to prepare were lacking. Providing medical students with appropriate and readily available resources to prepare may maximize their experiences during surgery observerships.

\section{PA - 2 - 8}

\section{Exploring the impact of early pre-clerkship} surgical exposure and mentorship on the perceptions of medical students towards surgical career stereotypes: A qualitative
analysis

Sylvie Bowden* University of Toronto, Shawn Khan* University of Toronto, Kimia Sorouri University of Toronto, Stephanie Searle University of Toronto, Lauren Carr University of Toronto, Jory Simpson University of Toronto

Background/Purpose: Surgeons are consistently viewed by medical students as uncompassionate, resolute, and individualistic. The Surgical Exploration and Discovery (SEAD) program is a two-week program that offers workshops, seminars, observerships, and mentorship opportunities to preclerkship medical students. The purpose of this study was to explore whether early surgical exposure could alter the negative perceptions of medical students towards surgeons and a surgical career.

Methods: This prospective cohort study included 30 preclerkship medical students (18 male, 12 female) who participated in the SEAD program at the University of Toronto during the summer of 2017. A constructivist grounded theory approach was used to qualitatively analyze six independent focus group interviews, conducted at both initiation and completion of the program.

Results: Focus group interviews suggested that students were more amenable to pursuing surgical careers after completing SEAD, despite acknowledging potential challenges. Students often 
compared their experience to previous single-day observerships and reported the two-week design of the program allowed them to better appreciate the surgical work-life balance. Students described surgeon mentors as kinder than expected, enthusiastic about teaching, and passionate about their work. Students viewed the lifestyle of surgery to be more favourable upon increased exposure to different surgical setups. In particular, female students were able to gain a better understanding of how to overcome female-specific challenges through female mentorship.

Conclusion: The SEAD program successfully contributed to changing negative perceptions about surgeons and a surgical career, and informed students of female-specific challenges and the capacity of surgeons to tailor their practice to achieve work-life balance. ${ }^{*}$ Co-first author 
PA - 3 - 1

\section{Scoping Review on Behaviour Remediation in Post-Graduate Medical Trainees}

Farina Rafiq Dalhousie University, Dr. Babar Haroon Dalhousie University

Background/Purpose: In the era of competency based education (CBE), behaviour remediation in post graduate (PG) medical trainees is highly relevant. Behaviour falls under "professionalism", an important component of the CanMeds framework as well as the Accreditation Council for Graduate Medical Education (ACGME) core competencies for physicians. Despite its importance, however, PG programs are unsure as to how to appropriately remediate trainees with deficiencies in these domains. Traditionally, trainees have been promoted within their training programs under the proficiency of medical expert with inadequacies in behaviour/professionalism. With a renewed emphasis on $\mathrm{CBE}$, and on patient safety and quality, there is a unique role for determining a method of remediation in PG medical trainees who have behavioural deficits.

Methods: A scoping review of behaviour remediation in PG medical trainees was conducted to determine mentoring strategies and interventions that have and have not been successful by training programs. PubMed and Embase electronic databases were searched.

Results: Out of the 92 articles found, 26 articles were included in this scoping review. Various strategies are implemented by PG programs to remediate PG medical trainees with behavioural/professionalism deficiencies. These strategies are listed in this review.

Conclusion: PG programs are unsure how to appropriately remediate PG medical trainees with behavioural/professionalism deficiencies. Based on our search we have listed various remediation strategies that are currently used by medical training programs, albeit inconsistently, and with questionable efficacy.

PA - 3 - 2
Challenges to the Integration of Entrustable Professional Activities into Clinical Clerkship

Zhubo Zhang Queen's University, Avrilynn Ding Queen's University, Andrea Winthrop Queen's University, Eleni Katsoulas Queen's University

Background/Purpose: Integration of entrustable professional activities (EPAs) within clerkship is part of the planned shift towards competency-based medical education in Canadian medical schools. We have previously identified discipline- and locationspecific differences in the level of supervision and performance of EPAs in clerkship at our institution. This follow-up study aims to understand the factors that contribute to those differences.

Methods: A focus group was conducted with the graduating 2018 class as part of a needs assessment aimed to assist in the planned integration of EPAs into clerkship. Directed questions were designed to stimulate discussion on the level of supervision and performance of EPAs. The narrative was transcribed verbatim and the transcript was coded independently between two reviewers. After final consensus, inductive analysis was used to identify patterns and themes.

Results: Six students participated in the one-hour focus group. Students reported certain factors that decreased the available opportunities for students to perform EPAs, such as a fast-paced clinical environment, a lack of consensus on the student role between sites, the specialization of a service, and the acuity of patient presentations. Students also elaborated on experiences that facilitated the performance of EPAs, including goal-setting at the start of a shift, prompting of students by preceptors, and the natural preceptor-student relationship that develops over time.

Conclusion: This study provides insight into the challenges in the implementation of EPAs in clerkship and identifies potential solutions that will assist in the planning of future clinical site and faculty 
development for successful shift to competencybased medical education.

\section{PA - 3 - 3 \\ What Does It Mean to Be Competent? An Inter-professional Definition in Anesthesia}

\author{
Natalie Lidster McMaster University, Caitlin \\ VanDeCappelle McMaster University, Saroo Sharma \\ McMaster University
}

Background/Purpose: Residency programs across the country are moving away from time-based training models toward competency-based programs. Competency refers to proficiency in technical dexterity, knowledge of anesthesia, and interpersonal skills. It is clear that a high level of capacity is desirable in these areas; however, is no universally accepted definition of competency exists as it relates to anesthesia. We propose that competence should be determined by those who work in the field of anesthesia using a definition where the expectations of our patients and clinical colleagues are valued and included.

Instructional Methods: We first performed a literature review of terms that we felt would inform us in our own understanding of competency; this also verified that no universally definition of competency existed. We then received ethics approval to carry out a qualitative research study using semi-structured interviews with 40 (ten in each cohort) patients, nurses, surgeons, and staff anesthesiologists. Participants were asked to share their perspective on competence and describe how that relates to their experiences and expectations with resident and staff anesthesiologists. Following this, interviews were transcribed and analyzed for emerging themes.

Target audience: Our objectives were: 1.) To determine the perspective of anesthesiologists, surgeons, nurses, and patients on competency in anesthesia and how this perspective compares and contrasts between roles 2.) To synthesize these perspectives into a cohesive, well-rounded concept of competency in anesthesia 3.) To describe how this concept can be applied to residency training programs to promote development of competent anesthesiologists
Summary/Results: We found several themes arose in participant interviews. First, the confidence of a trainee gave the appearance of competence, even though there was recognition that confidence and competence were distinct entities. Where it was found that a resident's actual ability did not match their perceived confidence, the resident lost credibility and was no longer seen as competent or trustworthy. As well, all participants described competence as being contextual. This meant that a trainee could be seen as competent in one setting but not be able to perform competently in a different set of circumstances; this pointed to the importance of varied and diverse learning opportunities. Second, competence in anesthesia referred to facilitation, meaning how well an anesthesiologist could facilitate a nurse's or surgeon's own tasks in being safe and patient focused, where anesthesiologists felt it was their own duty to be safe and patient focused in their own tasks. Third, patients measured the competence of their anesthesiologist using tangible measures closely linked to objective outcomes, for example absence of awareness and post operative analgesia. Lastly, there was universal recognition that competence was a spectrum composed of multiple components, but minimal proficiency in all domains was required to be considered wholly competent. This meant that communication and personal qualities were not underestimated.

Conclusion: In summary, we feel that competence is the ability to fulfil the duties of one's job and meet the expectations of those with whom we work and care for. Different professions will have expectations of competency based on how they view the anesthesiologist plays a role in supporting their own duties. Patients will have expectations based on their knowledge and previous experiences of anesthesiologists and we should expect this to be quite limited in some situations. Here, we should play an active role in education and preparation. Regardless of different expectations, our findings point to the significance of an inter-professional definition of competence being one that includes communication and leadership skills as well as qualities of understanding, compassion, and fairness.

\section{PA - 3 - 4}




\section{The use of simulation in emergency medicine UGME clerkship education: A QI Initiative}

Samuel Wilson University of Ottawa, Dalia Karol University of Ottawa, Christopher G. Elliott University of Ottawa, Kuan-chin Jean Chen University of Ottawa

Background/Purpose: Simulation is becoming widely adopted across medical disciplines and by different medical professionals. For medical students, emergency medicine simulation has been shown to increase knowledge, confidence and satisfaction. At the University of Ottawa Skills and Simulation Centre, third-year medical students participate in simulated scenarios common to Emergency Medicine (EM) as part of their mandatory EM clerkship rotation. This simulation curriculum was added in 2016, but has not yet been evaluated.

Methods: In groups of seven, medical students completed simulation sessions of the following: status asthmaticus, status epilepticus, urosepsis and breaking bad news. Student confidence with each topic was assessed before and after simulation with a written survey. Confidence scores pre- and postsimulation was compared with the Wilcoxon signed rank test.

Results: Medical student confidence with diagnosis of status asthmaticus ( $N=44, p=0.0449)$ and status epilepticus ( $N=45, p=0.0011$ ) increased significantly following simulation, whereas confidence with diagnosis of urosepsis was unchanged $(N=45, p=$ 0.0871 ). Treatment confidence increased significantly for status asthmaticus ( $N=47, p=0.0009$ ), status epilepticus ( $N=48, p=0.0005)$ and urosepsis ( $N=48$, $p<0.0001)$. Confidence for breaking bad news was not significantly changed after simulation $(\mathrm{N}=47, \mathrm{p}=$ 0.0689).

Conclusion: Simulation training in our EM clerkship rotation significantly increased the confidence of medical students for certain common EM presentations, but not for all. Further work will aim to understand why some simulation scenarios did not improve confidence, and look to improve existing scenarios.

\section{PA - 3 - 5}

\section{Developing a simulation curriculum to teach medical students to perform an ultrasound-guided needle insertion.}

Sachin Pasricha Queen's University, Zsuzsanna Keri Queen's University, Matthew Holden Queen's University, Tamas Ungi Queen's University, Gabor Fichtinger Queen's University

Background/Purpose: Undergraduate medical education curricula lack consistent training for ultrasound-guided procedures. We developed a simulation curriculum using augmented reality visualization to teach ultrasound-guided needle insertion on a vascular model to medical students. We measured performance and confidence learning curves.

Methods: Students participated in the 4-session simulation curriculum. Prior to and after each session, each student completed a simulated ultrasoundguided needle insertion on a vascular phantom model. Probe and needle motion was electromagnetically tracked to assess performance objectively using nine previously validated, computer-generated metrics, which were compared to expert benchmarks. Prior to each pre- and postsession test, students indicated their confidence on a scale of $0-10$. We performed signed rank tests to understand improvement from baseline, withinsession improvement, and after-session erosion, for both performance and confidence.

Results: 30 students participated. Performance improved from baseline by sessions 3 and 4 ( $p<0.01$, $p=0.04, p=0.01$, and $p=0.01$ when comparing baseline to pre-session 3, post-session 3, pre-session 3, and post-session 4, respectively). However, performance did not improve within individual sessions $(p=0.39$, $0.28,0.36$, and 0.42 for sessions $1-4$, respectively) or erode after individual sessions $(p=0.40,0.10$, and 0.25 after sessions 1-3, respectively). Confidence improved from baseline within session $1(p<0.01)$ and continued to improve within each session thereafter ( $p<0.01,0.01$, and 0.01 for sessions $2-4$, respectively).

Conclusion: The simulation curriculum yielded improvement in confidence earlier and more consistently than that in performance, raising concerns about single-session simulation curricula. 
PA - 3 - 6

Standardized patients no more: using nonstandardized patient stations in a clerkship objective structured clinical examination.

Vijay Sandhu University of Toronto, Raed Hawa University of Toronto, Carla Garcia University of Toronto, Andrea Waddell University of Toronto

Background/Purpose: The Observed Structured Clinical Exam (OSCE) has become the mainstay of clinical performance assessment in medical education. Centralization of OSCE assessment in a single high-stakes examination at our institution, and financial constraints necessitated re-evaluation of our specialty-specific OSCE. We wondered if we could introduce a lower cost version of our psychiatry OSCE circuit while maintaining sufficient rigor for an end of rotation exam.

Summary of the Innovation: We replaced one OSCE station with one Communicator Skills Assessment (ComSA) station. Based on stakeholder input, ComSA stations were designed to focus on obtaining collateral history and providing diagnosis and treatment specific education. Roleplays require minimal acting skills on the part of the examiner. We analyzed data from three iterations of a psychiatry clerkship examination $(n=126)$ that included four performance-based assessment stations ( 3 OSCE and 1 ComSA) and a written examination. A series of paired-samples tests of equivalence (TOST-P) determined that a ComSA station performed equivalently to an OSCE station in assessing content knowledge and communication skills. Furthermore, Cronbach's alpha omitted variable analysis demonstrated that a ComSA station had a similar impact on exam reliability to an OSCE station. Faculty and trainee feedback supported the ongoing use of the ComSA. The direct cost of the examination was reduced by $>20 \%$.

Conclusion: This study provides evidence for examiner roleplay stations in place of a traditional OSCE station. The ComSA was equivalent to the OSCE in evaluating the content and communication skills and inclusion of a ComSA station did not reduce exam reliability and improved feasibility.

\section{PA - 3 - 7 \\ Development of an Exercise During Pregnancy Online Learning Module for Family Medicine Residents}

Sophie Glanz University of Toronto, Rachel McDonald University of Toronto, Lisa Kachaniwsky University of Toronto, Sabrina Kolker University of Toronto, Pearl Yang University of Toronto, Karen Fleming University of Toronto

Background/Purpose: There are several health benefits of regular physical activity during pregnancy, including improving physical fitness, weight management and mood, and reducing the risk of gestational diabetes and hypertensive disorders of pregnancy. A scan of the literature suggests that implementation of guidelines for exercise during pregnancy is not taught consistently to family medicine residents. This project consists of creating an exercise during pregnancy online learning module, targeted for family medicine residents, to supplement the current postgraduate program. The objective of this online module is to familiarize family medicine residents with current exercise guidelines and promote confidence in prescribing exercise during pregnancy.

Summary of the Innovation: This e-module is part of a series of online maternity care learning modules, which includes Hypertensive Disorders of Pregnancy and Postpartum Care. The e-module includes validated evidence based tools such as the PARmed$X$ for Pregnancy addressing absolute and relative contraindications to exercise, videos demonstrating exercise counselling and implementation of a FITT (Frequency, Intensity, Time, Type) prescription, and safety tips. The module includes multiple choice questions as well as check box questions to test the learner's knowledge throughout the learning exercise.

Conclusion: By having this resource available to family medicine residents, knowledge and selfefficacy for prescribing exercise during pregnancy could potentially increase, contributing to delivering the highest level of evidence-based care to patients. 


\section{PA 3-8}

\section{How a medical students committee drastically changed the way to prepare for OSCE through peer education methods}

Vincent Ménard-Cholette Université Laval, Élizabeth Richard Université Laval, Julie Thériault Université Laval, Emily Wang Université Laval

Background/Purpose: OSCEs can be really stressful for medical students. Specifically, at an undergraduate level, students have very limited experience in clinical interviews. Moreover, they can lose themselves in the vast quantity of information to study, focus on very rare presentations or miss mustask questions or physical signs. All around, the challenges of OSCEs are known best by the students themselves through past successes and failures. A need for better tools and more practice was raised among students. New educational projects can be tedious and complicated to set up. It requires personnel, time, money and energy. Generally, medicine faculties are already trying their best to offer students the tools they need to succeed.

Summary of the Innovation: At Université Laval, a student committee, the Groupe de perfectionnement des habiletés cliniques (GPHC), which stands for group of improvement in clinical abilities, was created in 2010 to address this need. This group is focused on offering peer education and educational tools to medical students in addition to the medical program. The GPHC is now offering various workshops on clinical thinking. It also published a new book written by students and revised by physicians. Furthermore, the committee offers a web application allowing student to practice OSCE.

Conclusion: This student project successfully reached a structure allowing students to bring ideas and, through peer education, introduce innovative tools for medical students. This project shows how, with the support of the faculty, an independent committee thrived and developed reliable learning methods that became used by the vast majority of students. These methods helped students to assess their level of preparation and all around feel more prepared for their

OSCES.
PA 3-9

Using Self-Reported Measures of Confidence and Anxiety to Determine the Efficacy of the Surgical Exploration And Discovery (SEAD) Program in Reducing Anxiety and Increasing Confidence in Performing Procedural Skills

Frank Battaglia University of Ottawa, Emilie Langlois University of Ottawa, Marisa Market University of Ottawa, John Shin University of Ottawa, Christine Seabrook University of Ottawa, Tim Brandys University of Ottawa

Background/Purpose: Clerkship students feel increased anxiety and lack of confidence when it comes to surgery. This study assessed whether participation in Surgical Exploration and Discovery (SEAD), a two-week intensive surgical program that includes career information, simulation workshops and operating room observerships, would help decrease anxiety, increase confidence, and foster interest in a surgical career.

Methods: 30 first year medical students were randomly selected for the SEAD program and 32 were only given the program's instruction manual during the duration of the program serving as the control. At baseline and after the completion of SEAD, both groups were given a survey containing the State Trait Anxiety Inventory that measures self-reported anxiety levels with an adjunct that gauges confidence and interest in a surgical career.

Results: Students who participated in the program showed significant improvements in self-perceived knowledge and confidence for each surgical skill: scrubbing ( $p$-value $<0.001, \quad p$-value $<0.001$ ), maintaining sterility ( $p$-value $<0.001, p$-value $<0.001)$, and surgical assisting ( $p$-value $<0.001, p$-value $<0.001$ ). However, there was no difference in the average state anxiety with procedural skills ( $p$-value $=0.190$ ) between students who participated in SEAD and those who did not. Students who completed SEAD had a notable increase in their interest in pursuing a career in surgery compared to their pre-test $(p-$ value $=0.020$ ) and compared to the control group ( $p$ value $=0.600$ ). 
Conclusion: The SEAD program may increase medical students' confidence and interest in pursuing a surgical career. These results encourage offering medical students with similar opportunities that provide exposure to surgery in pre-clerkship. 


\section{Sunday, April 14th - 10:00-11:15}

Poster Group PA - 4

\section{PA - 4 - 1}

\section{Quick Refresher Sessions (QRS): improving chest compression training for medical students}

\section{Alexander Cormier Queen's University, Erin Brennan Queen's University}

Background/Purpose: High-quality CPR saves lives; however, current certification standards leave providers poorly prepared to perform effective chest compressions (CCS). We designed a training program based on the emerging model of skill maintenance through frequent short practice sessions. Our goal was to provide medical students with access to efficient and effective CC training.

Methods: Thirty-six second-year medical students were randomized to three groups that trained at different frequencies: once every two months (q2mth), once every four months (q4mth), and a control group that received no training. Our "Quick Refresher Sessions" (QRS) were completed using an autonomous manikin with the ability to provide realtime quantitative feedback on CC quality. Participants completed a one-minute pre-test, viewed a oneminute focused training video, engaged in two minutes of practice with real-time feedback, and completed a one-minute post-test. Self-reported preand post-training comfort performing CCs were also recorded.

Results: Performance parameters measured by the manikin were CC depth, rate, release between CCs, and hand positioning. A final "compression score" assessed integrated performance across these parameters. Immediately following training, subjects had significant improvement in compression score ( $p$ $<0.001$ ) and skill comfort ( $p<0.001)$. At eight months, both intervention groups, q2th and $q 4 \mathrm{mth}$, achieved higher compression scores than the control group ( $p=0.001$ and $p=0.011$, respectively) and showed greater increase in comfort levels across the study ( $p=0.002$ and $p=0.010$, respectively).
Conclusion: QRS training every two or four months led to improved CC quality and provider comfort with this life-saving skill.

\section{PA - 4 - 2 \\ Traditional versus 360-Degree Video- Modelling for teaching Central Venous Catheter placement using task trainers}

Evan Mah University of Saskatchewan, Megan Deck University of Saskatchewan, Julie Yu University of Saskatchewan, Kish Lyster University of Saskatchewan, Joann Kawchuk University of Saskatchewan, Brian Brownbridge University of Saskatchewan, Alison Turnquist University of Saskatchewan, Luke Terrett University of Saskatchewan, Brent Thoma University of Saskatchewan

Background/Purpose: Central Venous Catheter (CVC) placement is a common critical care procedure. Simulation-based training reduces iatrogenic CVCrelated complications. Video modelling (VM) is an instructional adjunct that improves the quality and success of CVC insertion. The immersive experience of 360-degree video recording has improved recall and skill translation in some contexts, however, its role in teaching medical procedures has not been established. We hypothesized that, relative to traditional VM, 360-degree VM would decrease cognitive load and enhance ultrasound-guided CVC insertion skill acquisition in novice residents.

Methods: Thirty-two resident physicians from anesthesiology, general surgery, emergency medicine, and internal medicine were randomized into traditional (control) or 360-degree VM (intervention) groups for three CVC training sessions. Skill acquisition was quantified by procedural time (needle puncture to tegaderm placement) and a 5point entrustment score (O-Score) completed by their supervisor. Cognitive load was quantified using the NASA Task Load Index (TLX). Mean ( \pm standard deviations) values were compared using two-tailed ttests. 
Results: Group demographics were similar. Entrustment scores improved significantly in both groups from the first $(3.3 \pm 0.8)$ to third $(4.1 \pm 1.1, p$ $<0.02)$ session. The control group $(4.6 \pm 0.7)$ received higher entrustment scores following the final session than the intervention group $(3.7 \pm 1.2, p<0.05)$. There was no significant differences between NASA TLX scores or procedural time.

Conclusion: The preliminary data suggests that there is no additional benefit to utilizing 360-degree video modeling as an instructional tool. Counter to our hypothesis, the group viewing traditional videos received significantly higher entrustment scores.

\section{PA - 4 - 4}

\section{A Pre-Clerkship Procedural Curriculum designed for the future of Canadian Medical Education: A Pilot and Feasibility Study}

Frank Battaglia University of Ottawa, Maria Merlano University of Ottawa, Céline Sayed University of Ottawa, Meghan McConnell University of Ottawa, Christopher Ramnanan University of Ottawa, Nikhil Rastogi University of Ottawa

Background/Purpose: Procedural skills training varies significantly across Canadian medical schools, and there is currently no standardized assessment tool to evaluate its benefits. This project aims to develop a curriculum that teaches 2 nd-year medical students to perform and evaluate procedural skills, while laying the foundation for Entrustable Professional Activities (EPAs). The goals of this program include decreasing anxiety, increasing confidence, and achieving competence for students and also allowing staff to judge the appropriate level of supervision when delegating learners to perform basic procedures in the team setting. Our curriculum incorporates, near-peer teaching as well as near peer formative assessment.

Methods: Each of the twelve 2nd year participants completed a State Trait Anxiety Inventory and selfreported confidence questionnaire related to procedural skills. Students participated in four sessions taught by expert physicians over a five month period. A new skill was taught at each monthly workshop and an opportunity to practice previously taught skills was provided. Skills were assessed in a skills integration simulation OSCE, and the anxiety and confidence questionnaire was repeated.

Results: Students who completed this pilot program showed a significant decrease in mean anxiety state (2.48 vs 1.74 , $p$-value $<0.001)$, while the control group did not ( $p$-value=0.408). When assessing confidence, students who completed this program showed increased self-assessed knowledge and confidence in each of the program's assessed skills. An increased level of competency was achieved in each skill by each student as assessed by the expert physicians.

Conclusion: This innovative event allows students to apply their knowledge of the social determinants of health to patient cases and integrate it with evolving history-taking skills. These sessions may help strengthen culturally appropriate clinical and communication skills among medical trainees, while enhancing population health teaching and health advocacy in undergraduate medicine.

\section{PA - 4 - 5}

\section{Program Assessment: Taking stock of the current state of Canadian Undergraduate Medical Education in Procedural Skills Curricula}

Frank Battaglia University of Ottawa, Meghan McConnell University of Ottawa, Christopher Ramnanan University of Ottawa, Nikhil Rastogi University of Ottawa, Maria Merlano University of Ottawa, Celine Sayed University of Ottawa

Background/Purpose: The objectives of the study were: (i) to characterize procedural skills education currently employed in pre-clerkship and clerkship curricula; (ii) to determine what skills physicianeducators think medical students should know upon graduation; and (iii) to identify physician-educator perceptions regarding the development of preclerkship procedural curriculum.

Methods: A web-based survey was distributed to 201 clinician-educators across Canada's 17 medical schools. Respondents were directed to an individualized survey based on their self-identified, roles at their institution. Respondents were asked demographic questions, what procedural skills are 
being taught and in what setting at their institution, and their opinions on the value of a pre-clerkship procedural curriculum.

Results: From the 17 school's surveyed, 8 schools responded "yes" that they had a clerkship procedural curriculum. For a pre-clerkship procedural curriculum, only 4 schools responded "yes". The top 10 procedurals skills identified that medical students should know upon graduation, in order, are: IV Access, Airway Management/Ventilator Management, Local anesthesia/field block, Casting, Spontaneous Vaginal Delivery, Testing for STIs, Phlebotomy, Suturing of Lacerations, Nasogastric Tube Placement, and Venipuncture. Clinicianeducators strongly supported a pre-clerkship procedural curriculum (median=4.00/5.00, mode $=5.00 / 5.00)$, and they believed it would decrease anxiety (median $=4.00 / 5.00$ ), increase confidence (median=4.00/5.00), and increase technical ability (median=3.00/5.00) in incoming clerks.

Conclusion: Across Canada, the state of UGME procedural skills education is inconsistent. With the identification of the Top 10 procedural skills medical students should know upon graduation, the learning objectives of a formal curriculum can be developed. With overwhelming support from both medical students and physician-educators alike, a formal preclerkship procedural curriculum is poised to redefine the landscape of procedural care for a whole new generation of physicians.

\section{PA - 4 - 6 \\ "Top points for bedside manner": Evaluating Inpatient Volunteer Satisfaction with Pre-Clerkship Clinical History and Physical Examination Training.}

Michael Elfassy University of Toronto, Laura Duncan University of Toronto, Alison Green University of Toronto, Katina Tzanetos University of Toronto, Joyce Nyhof-Young University of Toronto

Background/Purpose: Patient exposure is crucial to clinical competency training in medical education. Patient experiences when MD clerks play a direct role in patient care have been well studied. However, few studies have examined volunteer inpatients' (VIPs) experiences in pre-clerkship clinical training. This study investigated VIP experiences in bedside interactions with first year MD students in a clinical skills course where learners practiced basic competencies for educational, rather than patient care purposes.

Methods: English-speaking, adult inpatients from two teaching hospitals were consented following a clinical history and/or physical examination by first year MD students to complete a 10-minute, semi-structured interview on their recruitment, clinical interactions, overall experience, and satisfaction. Descriptive thematic analysis was used to characterize VIP insights.

Results: 93/97 VIPs approached agreed to participate. Preliminary analysis indicates that most VIPs felt that their interactions were positive; they felt listened to, respected, and able to contribute to medical education. Student factors contributing to positive VIP experiences included an ability to connect with patients as people via verbal/non-verbal communication expression compassion, patience and professionalism. VIPs recommended more informative recruitment processes to better understand, anticipate and prepare for their interactions. They also felt more comfortable when students appeared confident in their abilities.

Conclusion: Volunteering appears worthwhile and comfortable for VIPs. Recommendations for change are being processed to better inform preceptors, patients and students about expectations and best practices. Study results and lessons learned have value for clinical training in diverse health professions' training programs.

\section{PA - 4 - 7}




\section{Developing Skills for Developmental Disabilities in Preclinical Medical Students: Identifying How Adjuvant Clinical Electives can Impact Student Confidence}

Alexis Fong-Leboeuf University of Alberta, Irina Simin University of Alberta, Andy Le University of Alberta, Debra Andrews University of Alberta,

Background/Purpose: Medical students feel they require more training on patients with developmental disabilities (PWDD). Students at the University of Alberta developed a pre-clinical 12-hour module, "Developing Skills with Developmental Disabilities" (DSDD), to improve students' confidence regarding the care of PWDD.

Methods: Elective students, in addition to standard Neurosciences curriculum, received 6 hours of didactic teachings, and 6 hours of clinical experience at a rehabilitation hospital, where they attended a family interview and observed PWDD in a modified play/classroom setting. Control students participating in this study only received the standard curriculum. Elective students completed surveys administered on a 5-point Likert scale pertaining to self-perceived confidence pre- and post-elective. Controls completed the same surveys before and after the standard curriculum. Pre- and post-intervention scores were used to calculate relative improvement and then compared between groups via t-test.

Results: Data was collected from 2 consecutive years, including 35 DSDD students and 18 control students. Statistically significant ( $p<0.05)$ relative improvements were achieved in 4 of 10 self-reported scores pertaining to confidence interacting with PWDD, taking histories, recommending appropriate resources to families, and estimating developmental age.

Conclusion: DSDD appears to teach skills that may not be learned from the standard Neurosciences curriculum. In particular, interactions with children and families in an organized clinical environment appears to have provided the most benefit, as demonstrated by significant improvements in 4/10 learning objectives. Next steps would be to see if these changes translate into improved clinical skills in practice and ultimately better care for this population. 


\section{PB - 1 - 1 \\ Enquête de terrain sur la conformité des activités de DPC aux normes d'accréditation : un outil d'amélioration continue}

Cynthia Henriksen Université de Montréal, Vincent Jobin Université de Montréal, Louise Fugère Université de Montréal

Introduction: La Direction du développement professionnel continu (DDPC) de la Faculté de médecine de l'Université de Montréal (UdeM) est un organisme qui accrédite de nombreuses activités de DPC à travers le Québec. Différentes normes ont récemment été mises à jour, entre autres pour encadrer les relations avec l'industrie pharmaceutique et assurer des méthodes pédagogiques optimales. Objectif : L'objectif de l'enquête de terrain est d'obtenir des données dont le but est d'améliorer la qualité des formations, de faciliter le transfert des connaissances et d'acquérir des compétences.

Méthode: La DDPC de l'UdeM a mis en place un outil de vérification de la conformité aux règles éthiques et aux bonnes pratiques éducatives par les prestataires en DPC. L'ouitl permet de recueillir des données lors des activités et offre une rétroaction objective. La vérification est fondée sur une collecte systématique de données à propos des méthodes pédagogiques et vise l'adéquation aux normes éthiques, notamment le contrôle des biais potentiels, la gestion des conflits d'intérêts ainsi que le respect des droits d'auteurs.

Résultats: Les résultats de l'enquête témoignent d'un besoin d'amélioration dans les approches éducatives. Le contrôle des biais commerciaux et la gestion des conflits d'intérêts seront également à optimiser pour plusieurs prestataires en DPC. La planification et la mise en oeuvre de l'outil de vérification ont apporté une appréciation systématique de nos processus internes. Les résultats serviront de tremplin pour améliorer la qualité des activités offertes en DPC.
PB - 1 - 2

MD Program Teaching Awards of Excellence (MPTA): Celebrating pedagogical excellence in undergraduate medicine

Flora Jung University of Toronto, Mike Wiley University of Toronto, Frazer Howard University of Toronto, Nicholas Sequeira University of Toronto, Joyce Nyhof-Young The College of Family Physicians of Canada

Background/Purpose: Award programs that celebrate clinician-teacher quality communicate community appreciation and encourage pedagogical excellence. While many such programs exist at our medical school, they often recognize select few recipients and depend on nominators' initiative. MD students at the University of Toronto aimed to develop criteria for a novel faculty teaching award program that allows for the recognition of clinicianteacher excellence across the faculty without nomination.

Summary of the Innovation: Our MD Program Teaching Awards of Excellence (MPTA) process was student-initiated and faculty-approved. Focus groups were held with University of Toronto medical faculty and students to define MPTA criteria. Our university's Teaching Effectiveness Scores (TES) were the teaching quality measure; TES are calculated on a scale of 1-5 and equal the mean of medical student evaluations scores. All 2016-17 preclerkship teachers were eligible and stratified into the following three groups: small group facilitators ( $<25$ evaluations), lecturers ( $\geqq 25$ evaluations), and a combined pool. Award criteria identifying the top 10th percentile of teachers were recommended by faculty and student consensus. TES of $4.95 / 5,4.4 / 5$, and $4.9 / 5$ achieved this in the three groups. This identified 137/1483, $30 / 300$, and 178/1783 awardee teachers respectively. In the combined pool, $100 \%$ of awardees were small group facilitators.

Conclusion: MPTA flexibly identifies clinicianteachers who demonstrate teaching excellence without the need for nominations. As small group 
facilitators tend to receive higher evaluation scores compared to lecturers, it is recommended that they be stratified into separate award pools to promote more equitable award distribution.

\section{PB - 1 - 3 \\ Participant outcomes of student-run interprofessional health promotion services to vulnerable populations in Sudbury, Ontario}

Sarah Mavin Northern Ontario School of Medicine, Gayle Adams-Carpino Northern Ontario School of Medicine, April Kindrat Northern Ontario School of Medicine

Background/Purpose: In 2007 Health Canada provided funding to six professional groups for integrating and highlighting interprofessional education (IPE) standards that would become explicit in each profession's accreditation (i.e. medicine, nursing, occupational therapy, pharmacy, physiotherapy and social work) (Coffey, 2015). IPE occurs when learners of two or more health or social care professions engage in learning with, from, and about each other to improve collaboration and delivery of care. IPE has also been identified as a solution to the various challenges of health care access in rural settings (Hays, 2008). This research examines the effectiveness of IPE for community/patient-centered care as integrated within a student interprofessional group, Reach Accés Zhibbi (RAZ).

Summary of the Innovation: Developed by learners from the Northern Ontario School of Medicine (NOSM), RAZ is an interprofessional student-led group that provides health promotion services in conjunction with community partner agencies. The purpose of the health promotion service is to provide students with practical interprofessional experience while they directly serve vulnerable populations living in the downtown core of Sudbury, Ontario. Qualitative and quantitative data was collected from health promotion activities via formal surveys.

Conclusion: Results demonstrate a positive response from participants of RAZ health promotion events. RAZ recognizes a need to revise the research methods to gather more broad impressions of their events, continually reassess the needs of the participants, as well as evaluate both short and long-term outcomes. Furthermore, future research directions will examine the longitudinal outcomes for professionals who did or did not participate in an undergraduate IPE program, specifically RAZ.

\section{PB - 1 - 4 \\ An Unexplored Landscape - Intra- Professional Configurations and Patient Care Networks}

Katherina Baranova Western University, Jacqueline Torti Western University, Mark Goldszmidt Western University

Background/Purpose: Creation of a patient care plan requires understanding who on the healthcare team will be proving follow-up and ongoing care. Current teaching and system design emphasize the importance of the family physician as the healthcare hub for all patients. While the realities of practice often do not match this expectation - especially for the multi-morbid patient - it is unclear what the configurations of care for patients, especially those without a family physician, look like.

Methods: A narrative literature review was conducted on the topics of principal care providers, orphaned patients, specialist roles, and patient complexity. 51 references were identified, and these were subjected to a thematic analysis to identify key areas of knowledge and uncertainty in the care landscape.

Results: Four themes emerged from the literature: the distinction between principal and primary care; the role of the specialist; the growing complexity of patients; and the fluidity of care configuration from orphaned patients to patients wholly reliant on specialty care. There was a substantial body of literature in the 1990s exploring specialists providing principal or primary care, but little since. More recently, specialty-specific bodies of literature (e.g. nephrology, psychiatry) have begun to re-address the intersection between specialty and primary care.

Conclusion: Our findings suggest a far more complex landscape of intra-professional collaboration than is 
currently taught. Understanding this complexity can allow medical trainees and supervisors to better explore, with each patient, the configuration that will best meet their healthcare needs. Future research is needed to characterize the identified configurations and explore the dynamics of each.

\section{PB - 1 - 5}

\section{A Health Professional (HP) Student-Led Approach to Building Interprofessional Collaborative Practice (IPCP) Skills}

Maichael Thejoe University of British Columbia, Carrie Krekoski University of British Columbia, Christie Newton University of British Columbia

Background/Purpose: IPCP is required to work effectively in today's healthcare system. The literature suggests that healthcare providers need to be trained interprofessionally to practice collaboratively. $40-60 \%$ of HP students' learning takes place in the clinical setting. TLEF funds supported the development and implementation of a model to ensure that all HP students receive comparable IPCP learning opportunities regardless of location or timing of their clinical placements.

Summary of the Innovation: The development of the Student-led Interprofessional Learning (SIPL) model included a literature search, needs assessment, and an environmental scan to identify current initiatives, resources, and fundamental principles. Key pillars of SIPL are comparable experiences, student assessment, portability, evaluative ease, and sustainability. SIPL can be leveraged in pre-existing placements with minimal resources. Pre-placement modules and educational tools prepare students for their placement. A student-led appreciative inquiry or collaborative practice can be selected based on local context and preference. These modalities provide opportunities for teaching and assessment in HP core competencies. Students complete post-placement reflections to consolidate learning.

Conclusion: SIPL was piloted at 49 sites across BC, Alberta, and Ontario, with students from medicine and other HP disciplines. Outcomes were evaluated through student and preceptor focus groups, surveys, and stakeholder interviews. Our findings suggest SIPL is highly effective. Educational programs found SIPL efficient to deploy with minimal effort, and patientcentred. Students enjoyed SIPL compared to previous interprofessional training and gained numerous benefits though engagement with other HP trainees. SIPL resources are available and can be used by other HP programs.

\section{PB - 1 - 6 \\ Lost in translation: How informative are residents' responses to care team queries through a mobile-based text messaging system?}

Nicholas Sequeira University of Toronto, Tom MacMillan University of Toronto, Robert $\mathbf{W u}$ University of Toronto, Rodrigo Cavalcanti University of Toronto

Background/Purpose: On our inpatient wards, residents, nurses and allied-health professionals communicate through a mobile-based text messaging system. While this improves workflow, it can decontextualize complex issues compared with verbal communication. Furthermore, multitasking by residents may result in overly brief, uninformative responses, resulting in inadequate closed-loop communication. This study aimed to characterize essential components of informative responses and analyze resident responses to requests from the careteam.

Methods: Closed-loop communication requires a response that ensures the sender knows that the receiver understood the message. One thousand queries and responses were analyzed to generate a coding scheme based on this principle. These messages were used to create four tiers of response quality, ranging from 1 (poor) to 4 (excellent). Response quality was based on three components: 1. Acknowledgement and reply to each element within the query. 2. If action is requested, clear indication of whether action will be taken and timing. 3. If no action is taken, explicit reasons for not acting.

Results: Of 200 (20\%) randomly selected responses, $10.5 \%, 3.0 \%, 19.0 \%$, and $67.5 \%$ were in tier 1, 2, 3, and 4 , respectively. Of 126 responses at night or on the weekend, $10.3 \%$ were tier 1 . Of 110 messages 
where the resident was asked to take action, $18.1 \%$ of responses were tier 1 (no indication whether action will be taken).

Conclusion: Approximately $10.5 \%$ of resident responses to care-team requests are uninformative, potentially leading to miscommunications and preventable errors. Results from this study will be used as a framework to teach residents effective communication strategies.

\section{PB - 1 - 7}

\section{CHIUS: Examining the Impact of Student-} Run Clinics on Interprofessional Healthcare

\section{Students}

Mona Maleki University of British Columbia, Kelly Huang University of British Columbia, Heather McEwen University of British Columbia, Glenn Regehr University of British Columbia

Background/Purpose: Among health care students there are various formats through which interprofessional learning is taught. Our aim was to evaluate the impact of the student-run clinics (SRC) at the Community Health Initiative by University Students ( CHIUS) on student participants from
Pharmacy, Medicine, Nursing, Social work and Occupational Therapy.

Summary of the Innovation: Twenty-three participants in the CHIUS student-run clinic (SRC) at the University of British Columbia took part in two focus group interviews.A combination of open-ended and specific interview questions were used to explore the participants' learning from the SRC. Using the Grounded Theory approach, transcribed interviews were iteratively analyzed and questions were adjusted for subsequent focus groups as the analysis revealed certain themes. Three investigators coded the data individually, consolidated the themes, and developed explanation models for each theme.

Conclusion: The CHIUS SRC provide realistic patientcare experiences that are unique from didactic learning and building collective competence as well as individual students' repertoire of knowledge. Participating in interprofessional SRCs help students understand interprofessional roles without delineating scopes of practice, facilitating team work and breaking down outdated hierarchies. The result is that students were able to blur the boundaries of professional responsibilities and come together as a team to provide holistic care. 


\section{Sunday, April 14th - 15:30-17:00}

Poster Group PB - 2

PB - 2 - 1

\section{Implementing exercise as medicine into undergraduate medical curricula}

Irina Simin University of Alberta, Dhiren Naidu University of Alberta, Tracey Hillier University of Alberta

Background/Purpose: Physical activity (PA) is associated with decreased risk in major chronic diseases. Physicians have been identified as serving a key role in promoting PA to their patients. Physicianprovided PA counseling and exercise prescription has been shown to increase patients' PA levels. Insufficient educational opportunities have been cited as a primary contributor to the underprescription of exercise. The objective of this project was to review current medical education literature and curriculum to identify best practice for exercise education.

Methods: A scoping review to identify best practice in medical education on the topic of exercise was performed. Subsequently, our current curriculum pertaining to the inclusion of exercise education was reviewed, using key terms such as "exercise" and "PA".

Results: Three articles were identified that address the significance of exercise education for physicians. There were no studies identified on the efficacy of exercise curriculum in medical education. Learning objectives with regard to the importance and prescription of exercise were developed. Two lectures in total are currently delivered in the second year, within the Musculoskeletal block. The lectures were reviewed to identify gaps between the current state and the desired learning objectives.

Conclusion: Although literature exists emphasizing the importance of physician education on exercise prescription and referral, a review of our curriculum found that there is limited content and not all objectives were covered. Based on these findings, the content of existing lectures will be revised and an additional introductory lecture has been included in the curriculum to expand medical students' knowledge regarding exercise in medicine.

PB - 2 - 2

Health Policy and Healthcare System Sciences: An Essential Component of Medical Education

Tul-Zahra Rida University of Ottawa, Laura Muldoon University of Ottawa, Marie-Hélène Chomienne* University of Ottawa, Karine Toupin-April* University of Ottawa

Background/Purpose: Results of 2017 AFMC Graduation Questionnaires revealed that University of Ottawa (uOttawa) medical graduates rate their preparation in Health Policy and Healthcare System Sciences (HPHSS) as inadequate. This project aimed to understand how HPHSS are currently taught in uOttawa's UGME curriculum, and proposes a new, more comprehensive HPHSS curriculum.

Methods: An environmental scan of HPHSS curricula was conducted at five Canadian and one international medical schools. A literature review was performed to identify: 1) a validated framework to address gaps in uOttawa's HPHSS curriculum; 2) critical domains and themes essential to students' education; and 3) evidence-based modalities for content delivery and skills development in HPHSS. Relevant MCCQE learning objectives (LOs) were compared with uOttawa's LOs to identify gaps and address them.

Results: The environmental scan highlighted variability amongst HPHSS curricula across medical schools. Lack of a standardized Canadian HPHSS curricular framework was identified. Four contentbased domains (healthcare system \& process, policy \& principles, economics \& management, and system improvement), and seven skills-based domains (leadership, collaboration, inter-professional education, evidence based medicine and research, professionalism and ethics, scholarship, and physician-patient communication) were identified as essential HPHSS components. Previous longitudinal integration of HPHSS content in uOttawa's curriculum diminished its visibility, requiring HPHSS educational 
activities to be appropriately identified. Thus, $42.8 \%$ of uOttawa's HPHSS-related LOs were revised. New teaching sessions are being planned to address identified gaps.

Conclusion: This work identified curriculum gaps and LOs for modification at uOttawa. Addressing these gaps will help train socially responsible physicians with improved competency in HPHSS.

\section{PB - 2 - 3 \\ Designing a Medical Education Program to teach Pediatric Palliative Care to Health Care Professionals}

Chloe Thabet University of Ottawa, Dr. Megan Doherty University of Ottawa

Background/Purpose: Despite significant growth in the field of pediatric palliative care (PPC) in Canada, only $5-12 \%$ of children who could benefit from PPC are accessing these services. A recent survey found that over $60 \%$ of palliative care physicians were uncomfortable providing care for children. Providing PPC education may increase health care providers' (HCP) comfort in this area. Objectives: This study describes gaps in knowledge and training in PPC as perceived by HCPs and aims to characterize their educational needs using CIPP (context, input, process, product) analysis. These are first steps in designing and evaluating a program to teach PPC.

Methods: We used a mixed-methods approach which involved (1) a literature review, (2) a survey of stakeholders, and (3) establishing consensus using an expert panel.

Results: We identified 11 primary themes regarding gaps in PPC training from a review of (1) scientific and gray literature, (2) training objectives and competencies from relevant professional bodies and (3) existing palliative care training courses. We then conducted an online survey of $\mathrm{HCP}$, regarding the identified themes and training delivery methods. These findings were then reviewed by an expert panel to establish consensus about content, desired format, and educational methodologies. We identified a blended learning program consisting of 3 online modules combined with 1-day multi-professional workshop (8 modules), delivered by a specialized PPC team as the desired format for an education program in PPC.

Conclusion: The results of this study will be used to guide the development of a medical education program in PPC.

\section{PB - 2 - 4 \\ Student experiences with on-line modular patient safety and quality improvement curriculum}

Tatiana Fras University of Saskatchewan, Dr. Meredith McKague University of Saskatchewan, Dr. Regina Taylor-Gjevre University of Saskatchewan, Dr Michael Prystajecky University of Saskatchewan, Krista Trinder University of Saskatchewan

Background/Purpose: Second and fourth year medical students are now required to complete a selection of Institute for Healthcare Improvement (IHI) patient safety and quality improvement (PS/QI) courses. Gaining the perspectives of these students will assist with future planning of PS/QI curricula. We want to understand if an online learning tool is efficient and effective, and to explore students' perspectives on integrating course content with inprogram curriculum, and applicability to current or future practice.

Methods: This evaluation used on-line surveys and student focus groups. Two Focus groups (one with Year 2 students and one with Year 4 students) were held in April 2018, gaining qualitative data and feedback. The feedback from focus groups directed questions included on the standard course evaluation given at the end of Term 2.

Results: Surveys for specific curricular segments with an integrated narrative component yielded a response rate of $45 \%$. More than half of respondents recognize $P S$ and QI to be as an important concept in UGME, however students felt that the timing of the course may have been a better fit directly prior to clerkship and/or residency. Students enjoyed the online method of learning about PS/QI, however feedback around small-group learning and anecdotal lectures from healthcare professionals was received. 
Conclusion: Importing on-line curriculum content from established external organizations is an attractive option for medical schools, however determining how to best integrate such content within the curriculum presents a challenge. This evaluation will assist the $U$ of $S M D$ program in considering if, and how, such curriculum can be effectively incorporated.

\section{PB - 2 - 5 \\ Piloting Photovoice in a Student-Run Clinic: Implementing a novel reflection method with interdisciplinary healthcare trainees during service-based learning placements}

Fangyi Liu University of Toronto, Yang Qin University of Toronto, Imaan Javeed University of Toronto, Joyce Nyhof-Young University of Toronto

Background/Purpose: Photovoice, a communication method consisting of documentary photography and accompanying narrative captions, is an emerging curriculum tool for critical reflection in healthcare professional education. We present a student-run Photovoice project with interdisciplinary healthcare professional students (HPSs) completing a four-week volunteer community placement at IMAGINE Clinic, a student-run community health initiative providing health care to downtown Toronto neighborhoods. Purpose: (1) Explore Photovoice as a critical reflection method in interdisciplinary service-based learning. (2) Identify reflective themes of learners, advantages and challenges, and (3) gauge participant satisfaction with this curriculum approach.

Methods: Participants took weekly photographs during their placements, created accompanying captions, reflected on these outputs in two audiorecorded, facilitated group discussions, and completed a post-program satisfaction questionnaire. Transcripts underwent descriptive thematic analysis by 3 independent investigators and questionnaires were analyzed using basic descriptive statistics.

Results: Four HPS (nursing, medicine and pharmacy) in two cohorts took 27 photos, reflecting upon them in relation to their placements. Analysis to date identified six central themes - equity, mental wellness, power differentials, healthcare system, and healthcare professionals - indicative of two outcomes: empathy development and professional identity formation. Participants appreciated the flexibility of Photovoice reflection to accommodate personal reflection needs, describing their experience as "unique" and "deeply rewarding".

Conclusion: Photovoice appears not to have been previously used with interdisciplinary HPSs in servicebased learning. Results suggest critical reflection using Photovoice meets experiential service-based learning goals institutionally and may enhance professional and personal reflection in health professionals, particularly in the domains of empathy development and professional identity formation.

\section{PB - 2 - 6 \\ Discovery Healthcare: Promoting Interest in Healthcare Careers among High School Students from South-Western Ontario}

Vivian Tia Western University, Richard Yu Western University, John Cameron Western University, Dylan Russelo Western University, Danny Kim Western University

Background/Purpose: A major challenge that Southwestern Ontario (SWO) faces is the difficulty of healthcare worker recruitment. Discovery Healthcare (DHC) is a revamped summer camp that exposes high school students in SWO to medicine and other healthcare professions. The target demographic of this camp are student entering grade 10 as they are still deciding on their future career paths. These students are in the early stages of their schooling and can still make relevant academic choices in order to pursue a certain pathway. The goal of the camp is to get them interested in healthcare professions early on with the hope that they return to serve in their communities in the future.

Summary of the Innovation: Five-day long camps were held in July 2018 in Wingham, Sarnia, Chatham and Leamington. With the help of a community liaison, four medical students facilitated the camps. There was a total of 89 students, with an average of 22 students in each camp. Students ranged from grade $6-12$, but the majority had just completed 
grade 9. Students were introduced to various healthcare professions through local guest speakers, such as medicine, nursing, pharmacy and allied health. They learned clinical skills, including first aid, suturing, casting, and taking vitals. They were also introduced to case diagnosis, medical ethics and social determinants of health.

Conclusion: Surveys were distributed at the beginning and end of the camp to assess how the camp impacted the students' interest in the healthcare field and their level of understanding of a pathway to a career in healthcare. It was found that their interest level in pursuing a career in healthcare was slightly increased, while their level of understanding of the pathway to a healthcare profession was markedly increased. Overall the camp was very positively received, and the students appeared to have gained further insight into the everyday practices of their local healthcare professionals. DHC hopes to grow and continue to inspire more students from SWO communities to pursue healthcare careers.

\section{PB - 2 - 7 \\ Prescribing Competency in Undergraduate Medical Education}

\author{
Alison Bell Queen's University, Michelle Gibson \\ Queen's University
}

Background/Purpose: Graduating medical students may feel ill-prepared to prescribe medications safely. Recognizing this issue, Britain's Royal Pharmaceutical Society published a prescribing competency framework describing the outcomes effective prescribers should be able to demonstrate. Using this framework as a standard reference, gaps in medical undergraduate curricula can be addressed.

Methods: An environmental scan of our 2017-2018 undergraduate medical academic curriculum was conducted to identify learning events that addressed prescribing competency. Each learning event was matched to relevant competency subheadings within the prescribing competency framework. An analysis was carried out to identify gaps and redundancies.

Results: In the 2017-2018 academic year there were 54 curricular events dedicated to prescribing. 26 competency subheadings from the prescribing competency framework had multiple corresponding learning events, whereas 17 were not addressed in the curriculum. The competencies that were most frequently addressed tended to fall under "The Consultation" category. This includes Competency 1 "Assess the patient" and Competency 2 "Consider the options", the sole competencies in which all subheadings had at least one corresponding learning event. The majority of curricular gaps were noted among competencies grouped within the "Prescribing Governance" category.

Conclusion: This study has defined gaps and potential redundancies in the undergraduate curriculum at our institution. Further review will be required through feedback from curricular leaders, current students and recent graduates to develop resources and related assessments to address areas of concern. The approach used in this study and subsequent resources developed may be of use to other undergraduate curricula.

\section{PB - 2 - 8}

\section{"Are You Post-Call?": Promoting safe transport after call shifts}

Yasamin Mahjoub University of Alberta, Miranda Wan University of Alberta, Marie DeCock University of Alberta, Melanie Lewis University of Alberta

Background/Purpose: Provincial residents' associations in Canada have agreements with their respective faculties of medicine or health authorities to reimburse taxi rides after 24-hour call shifts. Medical students may work equivalent hours but no such safety measures exist.

Summary of the Innovation: We piloted a program for the 2019 clinical clerks at the University of Alberta (UofA) modeled after established taxi reimbursement programs. Students who felt unsafe to drive home after a 24+ hour call shift were encouraged to use taxi/rideshare services, then email their receipts and call schedule for reimbursement. Rides to the hospital prior to the shift, or to retrieve the vehicle were also eligible for reimbursement. The program was funded through the UofA Medical Students' Association \& Faculty of Medicine \& Dentistry Joint Funding program. This year, the initiative funded 44 rides for 
10 students (total cost $=\$ 769.82$ ). Rides were used on Internal Medicine, OB/GYN and General Surgery rotations. The average ride cost was $\$ 17.50$ $(S D=\$ 6.21)$. A survey conducted to collect feedback had the following results; 23 individuals responded, 7 $(30.4 \%)$ of whom had used the program. Of those surveyed, 21 (91.3\%) responded "agree or strongly agree" to the program promoting safety post-call. Areas for improvement identified included better promotion of the types of rides that were eligible for funding.

Conclusion: The "Are You Post Call" program successfully funded rides after call shifts for students at the UofA. This program can be expanded to other schools to promote safety for students.

\section{PB - 2 - 9 \\ What causes pediatric prescribing errors? Studying practice to guide education}

Richard Conn Queen's University Belfast, Orla Kearney Queen's University Belfast, Michael Shields Queen's University Belfast, Mary Tully University of Manchester, Tim Dornan Queen's University Belfast

Background/Purpose: What doctors are taught is more often based on 'speculation and ideology' than evidence. The 'wicked problem' of pediatric prescribing error exemplifies this. Lack of knowledge about causes means that educational solutions are failing to protect children from harm. As part of a doctoral research program that is generating educationally-relevant knowledge by investigating clinical practice, we systematically sought and synthesised evidence about causes of prescribing errors in children.

Methods: Using scoping review methodology, articles were obtained from systematic database searching, hand-searching reference lists, and opportunistic inclusion. We used an inclusive approach, accepting all evidence which contributed to understanding of how errors occur. We extracted and thematically synthesised causative factors. To add context and meaning to research evidence, we then conducted a 'return of findings' focus group with multidisciplinary stakeholders.

Results: Seventy articles showed six pediatric-specific causes of error: physiologic and pharmacokinetic factors; individualized dosing and calculations; medication formulations; off-license prescribing; communication with children and parents; and inexperience of pediatric practice. Multiple, contextually-influenced mechanisms interacted unpredictably to cause errors 'on the ground'.

Conclusion: This study reveals the distinctive challenges in prescribing safely for children, as practitioners contend with a complex array of pediatric-specific factors. Findings indicate that teaching foundational knowledge of pediatric pharmacology alone is insufficient. Education could address the specific error mechanisms identified within the review through targeted interventions and providing supervised opportunities to prescribe in context. Studying practice in-depth - including practitioners' experiences - is a vital step to ensure medical education delivers safe patient care. 
PB - 3 - 1

\section{Gender disparities in academic reference letters: A systematic review}

Shawn Khan University of Toronto, Abirami Kirubarajan* University of Toronto, Tahmina Shamsheri* McMaster University, Sangeeta Mehta University of Toronto

Background/Purpose: Reference letters comment on the qualities and capabilities of an applicant. For the Canadian Resident Matching Service (CaRMS) process, these recommendation letters are used to assess an individual's readiness to receive further medical training and practice as a physician. Despite their widespread use, reference letters can often be heavily shaped by stereotypes and unconscious biases rooted in gendered perceptions, rather than applicants' actual capacity for success in a given program.

Methods: We conducted a systematic review in accordance with Preferred Reporting Items for Systematic Reviews and Meta-Analysis (PRISMA) guidelines. We searched Embase, MEDLINE, and Psyclnfo from database inception to August 2018 for original studies that analyzed discrepancies in gender-linked adjectives in reference letters for academia or medical contexts. Study selection and data extraction were performed in duplicate, with a third author resolving discrepancies.

Results: A total of 15 studies were included from the 217 studies eligible for screening. In total, 6573 reference letters were analyzed from 5008 applicants. Out of the 4 studies that analyzed genderlinked adjectives, letters for women tended to systematically focus on communal rather than agentic traits. In comparison to male applicants, included studies noted a higher frequency of doubt raisers, personal life mentions, and comments about appearance for female applicants.

Conclusion: Reference letters for female applicants typically had a higher frequency of gendered language and doubt raisers. A better understanding of how gender is reflected within reference letters in medical education, and the subsequent impact on success rates, is needed. *Co-second authors

\section{PB - 3 - 2 \\ The Indigenous Program of the University of Sherbrooke Faculty of Medicine and Health Sciences}

Mélissa Nepton-Riverin Université de Sherbrooke, Genevieve Lavoie Université de Sherbrooke, Émilie Courtois Université de Sherbrooke, Pascale OuelletDufour Université de Sherbrooke

Background/Purpose: The University of Sherbrooke MD Program had historically little involvement with the Indigenous communities of Quebec. Some reasons for this include the lack of proximity to First Nations communities and the low proportion of Indigenous in the Quebec population (2\%). The establishment in 2006 of a distributed medical campus in the Saguenay-Lac-Saint-Jean region, comprising the Innu community of Mashteuiatsh and the Atikamekw community of Opitciwan, has given rise to the Sherbrooke Indigenous Program.

Summary of the Innovation: With the creation in 2008 of the Quebec First Nations and Inuit Faculties of Medicine Program, 11 Indigenous students have been admitted to the Sherbrooke MD Program since 2009, 2 at the Sherbrooke site and 9 at the Saguenay site. Educational activities about Indigenous history, culture and health issues have been introduced into the MD curriculum, as well as preclinical immersion experiences in several communities. Student interest groups emerged in both sites that have been active in cultural sensitization activities such as conferences, film projections, traditional retreats and ceremonies, as well as involvement with the Saguenay Native Friendship Center. Service activities such as the annual mini-med schools in Opitciwan and Saguenay have proved to be transformative student experiences.

Conclusion: The Sherbrooke Indigenous Program has graduated its first practicing family physician in 2018, and its activities have enhanced dialogue and a 
reciprocal relationship with several Indigenous communities of Quebec.

\section{PB - 3 - 3}

\section{Evaluating a Novel Integrated Clinical Skills Program for Undergraduate Medical Students}

Kaitlyn Lam University of Toronto, Ben Shachar University of Toronto, Fok-Han Leung University of Toronto, David Rojas Gualdron University of Toronto, Joyce Nyhof-Young University of Toronto

Background/Purpose: Integration in medical education has been rising in popularity as a model for curriculum design. The University of Toronto MD Program developed a novel Integrated Clinical Experience (ICE) Program to provide students with a holistic perspective of patient health and population needs through experiential learning and application of clinical skills. However, differences in the meaning and application of integration amongst programs have led to challenges in conducting valid and systematic program evaluation. This qualitative evaluation study aimed to better understand undergraduate medical students' experiences in the ICE Program to inform program improvements and demonstrate an integrated model of systematic program evaluation.

Methods: Using a realist evaluation approach, focus groups were conducted with first and second-year medical students in the University of Toronto MD Program to get a better understanding of how successfully integration of the ICE program components has been implemented. Data was transcribed and thematically analyzed by two independent investigators.

Results: All students reported positive experiences in the ICE program. Although most students were not explicitly aware of the ICE Program's integration objectives and components, upon reflection they all identified common themes between course components and recalled subconsciously integrating skills from various courses into their interactions with patients.

Conclusion: Students require more explicit explanations of integration objectives to achieve conscious recognition of the ICE program's integrated design. However, lack of recognition did not impede students' ability to integrate learning from various course components into their clinical skills acumen. Reflective practice may enhance their awareness and understanding of ICE integration objectives.

\section{PB - 3 - 4}

\section{Evaluation of the effectiveness of the Global Medical Student Partnership (GMSP) initiative in medical education}

Hannah Samuels University of Toronto, Vanessa Rojas Luengas University of Toronto, Arman Zereshkian University of Toronto, Paula Veinot University of Toronto, Ashna Bowry University of Toronto, Marcus Law University of Toronto

Background/Purpose: As the world becomes increasingly interconnected, it is imperative for medical students to have a sound understanding of global health in order to bridge health inequities within and between countries. The Global Medical Student Partnership (GMSP) aims to create an international network of medical students who share their local medical and cultural expertise, communicate and collaborate to solve common healthcare problems, and promote health advocacy.

Summary of the Innovation: Medical students in Canada are paired with international peers and meet monthly using online face-to-face platforms to discuss medically-related global health cases. They then review their findings with local GMSP peers and a faculty facilitator, enabling the group to gain a wealth of global health knowledge on each topic. Upon program completion, 26 of 32 (81\%) students completed a questionnaire (6-point Likert scales) to assess GMSP's impact. Thirteen also participated in one-on-one interviews. Descriptive statistics were calculated and thematic analysis conducted.

Conclusion: After participating in GMSP, students agreed or strongly agreed that they knew more about their healthcare system, healthcare practices abroad and how to solve complex health issues $(92 \%, 85 \%$, $73 \%$ respectively), and that international collaboration and communication skills were more important to them (92\%, 92\% respectively). 
Qualitative data showed that despite logistical challenges, many students would recommend implementing GMSP into the medical curriculum. Our findings suggest that GMSP is helpful in developing the CanMEDS competencies of the Royal College of Physicians and Surgeons of Canada (e.g., collaboration). This program may be an important addition to medical education.

\section{PB - 3 - 5}

\section{Using Think-Aloud Method to Evaluate \& Re-Design the University of Alberta MD Program Website}

Aashna Duggal University of Alberta, Thomas Jeffery University of Alberta, Dr. Joanne Rodger University of Alberta, Patrick Von Hauff University of Alberta, Dr. Tracey Hillier University of Alberta

Background/Purpose: University websites are one of the most effective tools for recruiting prospective medical students to their institutions. When websites are not updated in a dynamic academic environment, they can quickly become out-of-date and difficult to navigate. The University of Alberta's MD Program website disseminates information about its admissions processes, educational curriculum, and academic strengths. The MD Program is committed to recruiting and admitting diverse students, improving the navigation and content organization of its website as part of a broader recruitment and admissions strategy. To facilitate improvements in the MD program website, researchers used a think-aloud process to collect data during website usability testing.

Summary of the Innovation: Medical students $(n=6)$ and faculty and staff members $(n=7)$ were assigned a series of task-scenarios to locate information about the MD program using only the navigation sidebar on the InVision-designed website prototype. Participants were asked to use the "think aloud" method, a verbal protocol analysis used to understand internal cognitive process during the completion of each task.1 Results from the think-aloud were used to evaluate the organization and navigation of the website, the ease with which different topics could be found, improve page headings, and re-group content.
Follow-up questions provided insight on website content requiring further development.

Conclusion: The think-aloud method highlighted gaps in both organizational structure and content not previously identified on the MD program website. The think-aloud method is therefore an extremely valuable tool that can be used by University stakeholders to facilitate website improvements and enhance recruitment of prospective students to their MD program.

\section{PB - 3 - 6 \\ Perceptions around duty hour restrictions in residency: A qualitative study of online Canadian medical student discussion groups.}

Anahita Dehmoobad Sharifabadi University of Ottawa, Asif Doja University of Ottawa, Chantalle Clarkin Bruyère Research Institute, 43 Bruyère St. Ottawa, Ontario, K1N 5C8

Background/Purpose: Duty hour restrictions (DHR) were introduced in an effort to reduce burnout in medical residents. There is currently substantial variability in the allowable maximum duty hours for a medical resident in Canada. Online discussion groups may allow a unique insight into trainees' perceptions of DHR in residency.

Methods: We used an inductive qualitative approach to analyze 427 posts from 14 discussion threads in the Premed101 forum, posted from 2000-2018. Using content analysis, we identified key themes in the discussion threads. Data management was facilitated by NVivo 11.

Results: Several advantages and disadvantages of DHR were discussed in the forums. There were prominent discussions regarding knowledge acquisition and skills training in medical education, and how they require continued exposure over time; there was thus a concern regarding whether DHR could impact the development of competence. As a result, the majority of posts reflected the view that blanket rules regarding resident hours may not adequately meet the training needs of various specialties, with surgical specialties requiring more 
training time. Furthermore, posters discussed the multifactorial dimensions of trainee fatigue; they felt that it was more closely linked to the nature of the work being done rather than the number of hours worked. Lastly, posters felt that open dialogue around DHR was a positive measure towards acknowledging and addressing trainee wellbeing.

Conclusion: Online discussion groups have the potential to provide unique insight into $\mathrm{DHR}$ in Canada. These perceptions may ultimately provide a trainee perspective regarding the how DHR should be implemented nationally.

\section{PB - 3 - 7}

\section{Training Medical Students and Residents in the Use of Electronic Health Records: A Systematic Review of the Literature}

Akshay Rajaram Queen's University, Nimesh Patel Queen's University, Zachary Hickey Queen's University, Joseph Newbigging Queen's University, Brent Wolfrom Queen's University

Background/Purpose: As the adoption of electronic health records (EHRs) accelerates and as healthcare systems use advanced analytical techniques on stored information to improve patient care, future physicians will need knowledge and skills to work effectively within this ecosystem.[1,2] Although competencies have been outlined at the undergraduate and postgraduate levels, the scope of educational initiatives targeting knowledge and skill development in the use of EHRs is unknown.

Methods: We conducted a systematic review of the literature following PRISMA guidelines to identify educational interventions designed to equip medical students and/or residents with knowledge and/or skills related to a broad spectrum of activities in EHRs. We summarize and, where possible, synthesize the quantitative and qualitative results of formal evaluation of these initiatives.

Results: Our search strategy yielded 523 unique citations; 485 papers were excluded following screening of the abstracts. We reviewed 38 full texts and seven studies were included in the final data abstraction. Five studies focused on medical students, two studies focused on residents, and one included both groups. Curricular interventions ranged from a one-hour module introducing navigation of the local EHR to a longitudinal training program comprising experiential and didactic elements.

Conclusion: Despite the existence of a broad set of EHR competencies, this review reveals few curricular initiatives focused on training learners to engage with record systems for both individual patient care and population health improvement. Future work must build on existing initiatives and address barriers to educational activities in this area, including a shortage of physician educators with informatics expertise and resource constraints. 


\section{Sunday, April 14th - 15:30-17:00}

Poster Group PB - 4

PB - 4 - 1

\section{Project ECHO: Filling the Gap in Pediatric Palliative Care Training in India}

Emily Evans University of Ottawa, Megan Doherty Children's Hospital of Eastern Ontario

Background/Purpose: More than 98\% of children who need palliative care live in low- or middle-income countries where access to palliative care is extremely limited. Lack of education about palliative care has been identified as a significant barrier to improving access to palliative care. Project ECHO uses webbased videoconference sessions to train healthcare professionals. We developed Project ECHO Pediatric Palliative Care (PPC), which focuses on training health professionals in South Asia to provide palliative care for children.

Summary of the Innovation: We developed the content and structure of our ECHO program by reviewing the literature, a web-based survey of stakeholders, and consultation with experts. Project ECHO PPC consists of a 90-min session occurring regularly every 2 weeks. On average, there are 34 participants, who join from 15 different health centres in India and Bangladesh. Video recordings and key learning points are sent to participants after each session. The sessions format is:

- Welcome and introductions (10 mins)

- Didactic teaching (30 mins) - Questions (10 mins)

- Case presentation (10 mins) - Case discussion (25 mins)

- Summary (5 mins)

The goal is to create a virtual learning community where participants can reflect and engage to deepen their knowledge of PPC. The use of discussions is intended to encourage deeper engagement with the material, and collaboration with colleagues. We have conducted baseline evaluations of the program, with plans for future evaluation.
Conclusion: Project ECHO PPC is an innovative educational format for delivering pediatric palliative care education in resource-limited sessions, which can bring together content experts and practitioners in a collaborative online community.

\section{PB - 4 - 2}

Pilot Project: Implementation of Deaf Cultural Competency Training in the McGill Faculty of Medicine's Undergraduate Medical Curriculum

\section{Aselin Weng McGill, Brian Tran McGill}

Background/Purpose: The phenomenon of Deaf culture is little known to medical students and physicians. The lack of Deaf Culture Competency (DCC) training leads to poor access to healthcare for Deaf patients. The aims of this project are three-fold: (i) assess the need for DCC training in medical school, (ii) evaluate the comfort of medical students on the topic and (iii) assess the efficacy of a 1-hour long interactive lecture in addressing this issue.

Summary of the Innovation: The study population was composed of second-year medical students at McGill University. 23 participated in the 1-hour long interactive lecture, the content of which included myths and misconceptions of Deaf people, the rights of Deaf patients, legalities around sign language interpreting, communication skills, and basic American Sign language instruction. Knowledge on DCC was assessed before and after the lecture in order to evaluate the efficacy of the intervention.

Conclusion: In the intervention group, there was a significant increase in the number of correct responses to the majority of the knowledge assessment questions (20 out of the 39) after the DCC interactive lecture. There is a significant increase in the total knowledge assessment score after the DCC interactive lecture (pre-intervention mean score: 23.4/39, post-intervention mean score: $32.3 / 39, p$ $<0.01$ ). The 1-hour long interactive lecture was designed to address the major topics of Deaf patient 
care and has shown to be effective even when compared to lengthier DCC programs previously described in the literature. The shorter duration of our interactive lecture renders it more implementable in medical curricula across the country.

\section{PB - 4 - 3 \\ Does personality matter? Perceptions of introverts in general surgery}

Victoria Luong Acadia University, Chris Shields Acadia University, Allison Petrie Acadia University, Katerina Neumann Dalhousie University

Background/Purpose: Recent studies draw attention to the pressures surgical residents feel to conform to surgical culture, with surgeons being historically described as aggressive, dominant, and decisive. Little is known about how introverts - who tend to be more quiet and reserved - fare in this environment and adapt to it. This study explored how introverts experience, and are perceived in, general surgery.

Methods: Purposively sampling by personality, we invited 6 general surgeons and 10 general surgery residents to complete personality questionnaires and individual semi-structured interviews. A constructivist grounded theory approach was used to uncover perceptions of the workplace and of introverts' strengths/challenges within it, as well as the consequences of a mismatch between culture and personality.

Results: Two distinct narratives were identified. The participants who felt that "personality doesn't matter" saw few differences in how introverts and extraverts experience surgery. However, those who felt that "personality matters" thought that, while both can be successful, introverts appear less assertive, confident, and decisive than extraverts. They believed that maintaining control of acute situations requires a loud, dominant leader and that introverts need to act more extraverted at work. Extraverts had initially pursued surgery because they saw themselves in its extravert stereotypes, while many introverts were initially deterred from surgery because they did not.
Conclusion: Introverts may face challenges in surgery due to an extravert-dominant culture; however, introverts can adapt to the specialty and bring strengths to it. Students should have the confidence to pursue specialties in which they don't initially seem to "fit."

\section{PB - 4 - 4}

\section{A Systematic Review of Online Academic Resources: Renal and Genitourinary}

Andy Grock, Anuja Bhalerao University of Toronto, Teresa Chan McMaster University, Brent Thoma University of Saskatchewan, Seth Trueger

Background/Purpose: Online resources available for emergency medicine (EM) trainees and physicians have variable quality and inconsistent coverage of core topics. A systematic way to comprehensively identify, collate, and audit online content is needed. We describe the application of a methodology to identify these resources for inclusion in topic-specific modules.

Methods: A list of module topics and related terms was generated from the American Board of Emergency Medicine's Model of the Clinical Practice of Emergency Medicine (MCPEM). The authors selected 'Renal and Genitourinary' for the first module, which contained 35 terms; MeSH headers and colloquial synonyms related to the topic and related terms were searched within the top 100 websites ranked by the Social Media Index and the GoogleFOAM customized search engine. Duplicate entries, journal articles, images, and archives were excluded. The quality of each article was rated using the revised METRIQ ( $r M E T R I Q$ ).

Results: The search yielded 13,058 online resources; 12,707 were excluded leaving 351 for review. A majority $(n=31 ; 89 \%)$ of the topics were covered by a resource. The median rMETRIQ score was 11/21 (IQR $8-14)$. Calculus of urinary tract was most prominently featured with 61 posts. 13 of 35 topics (37\%) had $<5$ posts.

Conclusion: We demonstrated the feasibility of systematically identifying high quality resources in an EM topic area. Some topics were overrepresented while others were not covered. A module constructed 
from this curated list of resources may guide trainees, teacher recommendations, and resource producers. Further entries in the series will address other topics relevant to EM.

\section{PB - 4 - 5 \\ Fostering adaptive expertise: exploring guideline use in mental health care}

Natasha Tang Queen's University, Maria Mylopoulus University of Toronto, Sanjeev Sockalingam University of Toronto

Background/Purpose: Clinical practice guidelines are often used to influence provider decisions in clinical care through the acquisition and application of evidence-based knowledge. Research in adaptive expertise, however, has demonstrated that physicians must be able to navigate between direct, efficient application of knowledge in routine situations and developing new solutions when handling non-routine cases. Expecting physicians to simply apply guidelines to their existing practice may be an oversimplification of a complex process. Thus, our qualitative pilot study explored how clinical guidelines might contribute to both routine and nonroutine care and how psychiatrists recognized cases that required variations from guidelines.

Methods: We conducted semi-structured interviews with 7 psychiatrists at an academic centre in Canada, exploring use of depression guidelines their practice. Analysis of the transcribed interviews was consistent with a constructivist grounded theory approach and utilized theories of adaptive expertise as a conceptual framework for the interpretation of the data.

Results: We identified three themes: 1) experts perceived guidelines as a flexible resource for clinical practice, underlying management strategies for both routine and non-routine care, 2) experts recognized non-routine cases based on multiple factors, including medical comorbidities, social context, personality traits and the trajectory of the depression, and 3) experts integrated many forms of knowledge for clinical decision-making in non-routine cases.

Conclusion: The results of this study contribute to the current discussion regarding the use of guidelines in clinical practice, and represent further research on how the framework of adaptive expertise informs understanding of problem-solving in clinical care.

\section{PB - 4 - 7}

\section{Virtual Doc: A virtual reality simulation teaching paediatric cardiopulmonary resuscitation}

Janaya E. Perron University of New South Wales, Michael J. Coffey University of New South Wales, Andrew Lovell-Simons University of New South Wales, Zheyu Li University of New South Wales, Seiya Takeda University of New South Wales, Luis Dominguez University of New South Wales, Chee Y. Ooi University of New South Wales

Background/Purpose: There is limited evidence for the use of virtual reality simulation in paediatric medical education. Therefore, we developed a virtual reality simulation, Virtual Doc (VD), that teaches paediatric cardiopulmonary resuscitation skills to medical students. This study evaluates the usability and perceived educational value of VD.

Methods: We recruited medical students from The University of New South Wales (Australia) through voluntary convenience sampling. Participants attempted at least one full VD case and completed two mixed-methods questionnaires. Survey 1 assessed game components using 3-point Likert-scale questions. Survey 2 evaluated educational validity using 3-point Likert-scale and dichotomous or trichotomous questions. The responses were analyzed using descriptive statistics.

Results: Twenty-six students were recruited and completed Survey 1 ( $n=24)$ and/or Survey $2(n=23)$. VD was seen as 'very', 'moderately' or 'not' consistent with real-world clinical experiences by $25.0 \%, 58.3 \%$ and $16.7 \%$ of respondents, respectively. $58.3 \%, 37.5 \%$ and $4.2 \%$ of participants were 'completely engrossed', 'mildly involved' or 'not involved' in the VD environment, respectively. In terms of gameplay, $69.6 \%$ and $73.9 \%$ of participants agreed that it was easy to understand how to play the video-game and found the gameplay elements useful in understanding cardiopulmonary resuscitation, respectively. $69.6 \%$ of participants agreed that VD 
improved their understanding of cardiopulmonary resuscitation. VD was enjoyed by $91.3 \%$ of participants, whereby $73.9 \%$ and $65.2 \%$ of participants would recommend this simulation to a colleague or friend, respectively.

Conclusion: Our findings demonstrate a positive response to VD. We plan to further investigate the efficacy of VD with a randomized controlled trial.

\section{PB - 4 - 8}

\section{VR Plexus: Can virtual reality on a smartphone improve neurology learning amongst medical students?}

Maxwell Ng McMaster University, Rohit Malyala McMaster University, He Tian Chen McMaster University, Katherine Kuo McMaster University, Alexander K. Ball McMaster University, Michel P. Rathbone McMaster University, Bruce Wainman McMaster University

Background/Purpose: There is increasing interest in the application of immersive stereoscopic virtual reality (VR) technologies to medical education. However, many studies on VR education assess costly VR equipment not easily accessible to students. Furthermore, there are no studies investigating the use of VR in teaching physiological concepts, such as neurology. By leveraging animation, a VR learning tool offers learners the opportunity to build upon knowledge gained from lecture or cadaver-based instruction, by illustrating a dynamic body system operating in a realistic 3D context.

Summary of the Innovation: We have developed a lightweight stereoscopic virtual reality (VR) application to facilitate learning of clinical neurology for undergraduate medical students, using the smartphone-compatible Google Cardboard headset. Our learning tool illustrates functional concepts such as the consequences of nerve lesions. We selected the Google Cardboard headset for its low cost and intuitive design, which allows it to be easily deployed in a course context.

Conclusion: We have demonstrated that it is possible to build a financially-accessible smartphone VR learning tool for teaching neuroanatomy and physiology. We collected and analyzed qualitative feedback from medical students to inform the further development of this learning tool. These improvements will be implemented and quantitatively assessed in an upcoming RCT trial, investigating the effect of VR on learning and retention of neurology concepts. This educational project provides insight regarding the design and use of VR in anatomy and physiology teaching. 